\title{
A short contemporary survey on pathological brain detection
}

\author{
Mengmeng Yang ${ }^{1,2, *}$, Yongyan Jiang ${ }^{3, *}$ \\ ${ }^{1}$ School of Computer Science and Technology, Nanjing Normal University, Nanjing, Jiangsu 210023, \\ China \\ 2 Jiangsu Key Laboratory of 3D Printing Equipment and Manufacturing, Nanjing, Jiangsu 210042, \\ China \\ ${ }^{3}$ College of Science, Zhongyuan University of Technology, Zhengzhou, 450007, China \\ Email: 1320981274@qq.com,jiangyongyan@126.com
}

Keywords: magnetic resonance imaging (MRI); pathological brain detection; classification

\begin{abstract}
Brain disease detection still is the hotspot and difficulty in the present society. Nevertheless, the emergence of magnetic resonance imaging (MRI) technology can be more convenient for the diagnosis of brain diseases. In this paper, we introduce several methods to detect pathological brain automatically, and analyze their advantages and disadvantages as well as to the direction of the future.
\end{abstract}

\section{Introduction}

Developing a pathological brain detection system (PBDS) is the tendency of social progress. With the comparison to the traditional method, it can help doctors to detect disease for patients accurately [1, 2]. Meanwhile, the patient can quickly get the pathological findings so as to get treatment.

Magnetic resonance imaging (MRI) [3-6] is a type of tomography. Its mechanism is based on magnetic resonance phenomena. Using MRI, the scanner can attain EM (full name is electromagnetic) signals [7-9]. Afterwards, the algorithm can reconstruct the inner image among human structure. It is suitable for almost diseases of the system, such as cancer, inflammation and a variety of congenital diseases [10-15]. The most important thing is that it does not contain any radiations [16-18].

In this paper, we will introduce some approaches for detecting pathologic brain disease and analyze the advantages and disadvantages.

\section{Methods and Results}

Lu (2016) [19] proposed a radial basis function neural network (RBFNN) to classify the brain images, Meanwhile, they compared its method with following five methods: (i) BPNN; (ii) DWT+PCA+SVM; (iii) WE+NBC; (iv) RT+PCA+LS-SVM; (v) RBFNN. The results showed that the accuracy of WE+RBFNN is higher than other methods.

Yang (2016) [20] proposed wavelet-energy to reduce the dimension of features. Then, they used the support vector machine $(\mathrm{SVM})$ as classifier, meanwhile, this paper employed the biogeography-based optimization (BBO) to find the most suitable parameters, i.e., $\sigma$ and $C$. Further, the accuracy, sensitivity, specificity and precision of this method achieved $97.78 \%, 98.12 \%, 92 \%$, and $99.52 \%$, respectively.

Zhang (2016) [21] employed 2-level stationary wavelet entropy (SWE) to extract feature, then they compared three methods: (i) the decision tree, (ii) $k$-nearest neighbors (shorted as KNN), (iii) support vector machine (abbreviated as SVM). The authors found that KNN performed the best among three classifiers. Therefore, they proposed "SWE + KNN" to detect multiple sclerosis (MS).

Zhou (2016) [22] proposed "WE+FNN" to classify a MR brains image as normal or abnormal. They made tests based on 1-5 decomposition levels to find which leads to the highest accuracy, the results showed that first-level decomposition achieves $100 \%$ accuracy and its only contains four 
features. However, this experiment used 64 images which it is very small.

Wang (2016) [23] designed a novel approach via an effective feature-fractional Fourier transform (FRFT) and a traditional feature reduction method-principal component analysis (PCA) to detect unilateral hearing loss. This paper employed a single-hidden-layer feed-forward neural network (SFNN) as classifier and used the classical Levenberg-Marquardt algorithm to train SFNN.

Wang (2016) [24] selected 28 Alzheimer's disease (AD) and 98 healthy controls (HCs) from OASIS dataset in order to develop an AD detection system. This system included wavelet entropy, multilayer perceptron (MLP) and biogeography-base optimization (BBO). Thereinto, the BBO was used to train MLP, in addition, experiments showed that BBO performed better than other swarm intelligence based training algorithms.

Chen (2016) [25] proposed "DWT+PCA+GEPSVM (with Tikhonov regularization)” to detect sensorineural hearing loss (SNHL). Experiment showed that the total accuracy achieved 95.71\% when the threshold in PCA was set to 99\%.

Sun (2016) [26] employed two-dimensional (2D) discrete wavelet transform (DWT). Their chose Haar wavelet. The authors used a three-level decomposition. Finally, the obtained 10 subbands.

In the classification stage, they employed the support vector machine with different kernels (KSVM). They tested the performances of different kernels. A new algorithm: quantum-behaved particle swarm optimization (abbreviated as QPSO) was used to train KSVM. In the experiment, their simulation showed that the accuracy achieved $98.22 \%$, the precision achieved $99.52 \%$, the specificity was $92.00 \%$, and the sensitivity was $98.59 \%$.

Yang (2016) [27] proposed a dual-tree DWT in order to improve the directional selectivity impaired by DWT. For an $m$-level decomposition, they extracted $12 m$ variance and entropy (VAE) features based on the coefficients of DTCWT. Further, this paper used SVM, generalized Eigenvalue Proximal SVM (GEPSVM), twin SVM (TSVM) as classifier. The results of experiment show that "DTCWT-VAE-TSVM" was superior to "DTCWT-VAE-SVM/GEPSVM" and other 19 contemporary methods. However, this approach also had limitations: the size of dataset was too small.

Zhang (2016) [28] put forward fractional Fourier entropy (FRFE) and multi-layer perceptron (MLP) in order to develop a novel pathological brain detection system (PBDS). This paper proposed two improvements including pruning technique (PT) and Biogeography-based optimization (BBO). Meanwhile, this paper introduced and made comparison among 3 pruning methods: (1) Kappa coefficient (KC); (2) dynamic pruning (DP); and (3) Bayesian detection boundaries (BDB).

Siddiqui (2015) [29] tested more than 300 subjects. The scans are of both T1-weighted and T2-weighted. Further, its method improved the efficiency of feature extraction significantly by more than 70 percentage. Their method improved dimension reduction and classification slight by less than 5 percentage.

\section{Discussion and conclusion}

In this paper, we introduce several methods to classify a brain image as abnormal or normal. Furthermore, we summarized the characteristics of these methods in Table 1. In the future, we shall discuss smart detection on other parts (not brain), such as breast [30, 31], lung, etc. 
Table 1 Comparison of several classification algorithms

\begin{tabular}{|c|c|c|c|}
\hline Author & Method used & Limitation & Accuracy \\
\hline$\frac{\mathrm{Lu} \quad(2016)}{[19]}$ & $\begin{array}{l}\text { 2D discrete wavelet transform } \\
\text { (DWT) was used to extract features, } \\
\text { wavelet-entropy (WE) to reduce the } \\
\text { dimension of features, RBFNN }\end{array}$ & $\begin{array}{l}\text { The dataset only contains } 125 \\
\text { images. }\end{array}$ & $95.44 \%$ \\
\hline$\frac{\text { Yang (2016) }}{\text { [20] }}$ & WE-SVM-BBO & $\begin{array}{l}\text { The brain dataset contains } 90 \\
\text { images which is too small. }\end{array}$ & $97.78 \%$ \\
\hline$\frac{\text { Zhang }}{(2016)}[21]$ & $\begin{array}{l}\text { SWE, KNN, } 10 \text {-fold cross validation } \\
\text { to prevent over-fitting. }\end{array}$ & $\begin{array}{l}\text { KNN needed to calculate the } \\
\text { distance } \mathrm{k} \text { times in the } \\
\text { validation stage. }\end{array}$ & $97.94 \%$ \\
\hline$\frac{\text { Zhou (2016) }}{\text { [22] }}$ & $\begin{array}{l}\text { 2-level 2D DWT, Wavelet entropy } \\
\text { (WE), FNN as classifier. }\end{array}$ & $\begin{array}{l}\text { The dataset only obtains } 64 \\
\text { images. }\end{array}$ & $100 \%$ \\
\hline$\frac{\text { Wang }}{(2016)}$ [23] & $\begin{array}{l}\text { fractional Fourier transform (FRFT), } \\
\text { PCA, SFNN }\end{array}$ & $\begin{array}{l}\text { This experiment only } \\
\text { contains } 49 \text { subjects. }\end{array}$ & $95.1 \%$ \\
\hline $\begin{array}{l}\text { Wang } \\
(2016)[24]\end{array}$ & $\begin{array}{l}\text { WE, multilayer perceptron, } \\
\text { biogeography-base optimization }\end{array}$ & $\begin{array}{l}\text { Wavelet-entropy is low } \\
\text { efficiency compared with } \\
\text { other methods, such as scale }\end{array}$ & $92.40 \%$ \\
\hline$\frac{\text { Chen (2016) }}{[25]}$ & $\begin{array}{l}\text { DWT was applied to decompose } \\
\text { brain images and used the PCA to } \\
\text { reduce the dimension of features. }\end{array}$ & $\begin{array}{l}\text { The sensitivity of this method } \\
\text { is lower than } \\
\text { "WPD+LS-SVM" in } S_{2} \text { and } \\
S_{3}\end{array}$ & $95.71 \%$ \\
\hline$\frac{\text { Sun }(2016)}{[26]}$ & DWT-KSVM-QPSO & $\begin{array}{l}\text { one Lyme Encephalopathy } \\
\text { image was wrongly classified } \\
\text { as normal }\end{array}$ & $98.22 \%$ \\
\hline$\frac{\text { Yang (2016) }}{[27]}$ & $\begin{array}{llr}\text { proposed and compared three } \\
\text { different classifiers based on }\end{array}$ & $\begin{array}{l}\text { the dataset does not reflect } \\
\text { the actual situation }\end{array}$ & $99.57 \%$ \\
\hline $\begin{array}{l}\text { Zhang } \\
\text { (2016) [28] }\end{array}$ & $\begin{array}{l}\text { DTCWT-VAE } \\
\text { fractional Fourier entropy (FRFE) to } \\
\text { extract } 12 \text { features for each brain } \\
\text { image, multi-layer perceptron (MLP) }\end{array}$ & $\begin{array}{l}\text { This approach was only } \\
\text { applied to T2-weighted } \\
\text { images. }\end{array}$ & $99.53 \%$ \\
\hline$\frac{\text { Siddiqui }}{(2015)[29]}$ & $\begin{array}{l}\text { DWT, PCA, LS-SVM with radial } \\
\text { basis function (RBF), } k \text {-fold stratified } \\
\text { cross validation }\end{array}$ & $\begin{array}{l}\text { This approach can only } \\
\text { verified brain MRIs. }\end{array}$ & $100 \%$ \\
\hline
\end{tabular}

\section{Acknowledgment}

This paper is supported by Open Program of Jiangsu Key Laboratory of 3D Printing Equipment and Manufacturing (3DL201602).

\section{Reference}

[1] D'Angelino, R.H.R., et al., Detection of bovine leukemia virus in brains of cattle with a neurological syndrome: pathological and molecular studies. BioMed research international, 2013. 2013: pp. 425646-425646

[2] Yasmin, M., et al., Pathological Brain Image Segmentation and Classification: A Survey. Current Medical Imaging Reviews, 2014. 10(3): pp. 163-177

[3] Wu, L., An MR brain images classifier via principal component analysis and kernel support vector machine. Progress In Electromagnetics Research, 2012. 130: pp. 369-388

[4] Wu, L., Magnetic Resonance Brain Image Classification by an Improved Artificial Bee Colony Algorithm. Progress in Electromagnetics Research, 2011. 116: pp. 65-79

[5] Wang, S. and L. Wu, A novel method for magnetic resonance brain image classification based on adaptive chaotic PSO. Progress in Electromagnetics Research, 2010. 109: pp. 325-343

[6] Zhan, T., Pathological brain detection by artificial intelligence in magnetic resonance imaging scanning. Progress in Electromagnetics Research, 2016. 156: pp. 105-133 
[7] Yang, J., Preclinical diagnosis of magnetic resonance (MR) brain images via discrete wavelet packet transform with Tsallis entropy and generalized eigenvalue proximal support vector machine (GEPSVM). Entropy, 2015. 17(4): pp. 1795-1813

[8] Yu, D., et al., Exponential wavelet iterative shrinkage thresholding algorithm with random shift for compressed sensing magnetic resonance imaging. IEEJ Transactions on Electrical and Electronic Engineering, 2015. 10(1): pp. 116-117

[9] Dong, Z., Classification of Alzheimer disease based on structural magnetic resonance imaging by kernel support vector machine decision tree. Progress In Electromagnetics Research, 2014. 144: pp. 171-184

[10]Yang, J.Q., et al., A Novel Compressed Sensing Method for Magnetic Resonance Imaging: Exponential Wavelet Iterative Shrinkage-Thresholding Algorithm with Random Shift. International Journal Of Biomedical Imaging, 2016, Article ID: 9416435

[11] Yang, J.F. and P. Sun, Magnetic resonance brain classification by a novel binary particle swarm optimization with mutation and time-varying acceleration coefficients. Biomedical Engineering-Biomedizinische Technik, 2016. 61(4): pp. 431-441

[12]Liu, G., et al., Detection of Alzheimer's Disease by Three-Dimensional Displacement Field Estimation in Structural Magnetic Resonance Imaging. Journal of Alzheimer's Disease, 2016. 50(1): pp. 233-248

[13]Du, S., Alzheimer's Disease Detection by Pseudo Zernike Moment and Linear Regression Classification. CNS \& Neurological Disorders - Drug Targets, 2017. 16(1): pp. 11-15

[14]Nayak, D.R. Detection of unilateral hearing loss by Stationary Wavelet Entropy. CNS \& Neurological Disorders - Drug Targets, 2017. 16, DOI: 10.2174/1871527315666161026115046 (Online).

[15]Lu, S. and X. Qiu, A Pathological Brain Detection System based on Extreme Learning Machine Optimized by Bat Algorithm. CNS \& Neurological Disorders - Drug Targets, 2017. 16(1): pp. 23-29

[16]Chen, S., et al., Magnetic resonance brain image classification based on weighted-type fractional Fourier transform and nonparallel support vector machine. International Journal of Imaging Systems and Technology, 2015. 25(4): pp. 317-327

[17]Liu, A., Magnetic resonance brain image classification via stationary wavelet transform and generalized eigenvalue proximal support vector machine. Journal of Medical Imaging and Health Informatics, 2015. 5(7): pp. 1395-1403

[18]Dong, Z., et al., Exponential wavelet iterative shrinkage thresholding algorithm for compressed sensing magnetic resonance imaging. Information Sciences, 2015. 322: pp. 115-132

[19]Lu, Z.H., et al., A Pathological Brain Detection System Based on Radial Basis Function Neural Network. Journal of Medical Imaging and Health Informatics, 2016. 6(5): pp. 1218-1222

[20]Yang, G., et al., Automated classification of brain images using wavelet-energy and biogeography-based optimization. Multimedia Tools and Applications, 2016. 75(23): pp. 15601-15617

[21]Zhang, Y., et al., Comparison of machine learning methods for stationary wavelet entropy-based multiple sclerosis detection: decision tree, k-nearest neighbors, and support vector machine. Simulation-Transactions of the Society for Modeling and Simulation International, 2016. 92(9): pp. 861-871

[22]Zhou, X.-X., et al., Detection of abnormal MR brains based on wavelet entropy and feature selection. Ieej Transactions on Electrical and Electronic Engineering, 2016. 11(3): pp. 364-373

[23]Wang, S., et al., Detection of Left-Sided and Right-Sided Hearing Loss via Fractional Fourier 
Transform. Entropy, 2016. 18(5)

[24]Wang, S.-H. Single slice based detection for Alzheimer's disease via wavelet entropy and multilayer perceptron trained by biogeography-based optimization. Multimedia Tools and Applications, 2016, DOI: 10.1007/s11042-016-4222-4 (Online).

[25]Chen, Y., et al., Sensorineural hearing loss detection via discrete wavelet transform and principal component analysis combined with generalized eigenvalue proximal support vector machine and Tikhonov regularization. Multimedia Tools and Applications, 2016

[26]Sun, P., Preliminary research on abnormal brain detection by wavelet-energy and quantum-behaved PSO. Technology and Health Care, 2016. 24(s2): pp. S641-S649

[27]Yang, M., Dual-Tree Complex Wavelet Transform and Twin Support Vector Machine for Pathological Brain Detection. Applied Sciences, 2016. 6(6), Article ID: 169

[28]Zhang, Y., et al., A Multilayer Perceptron Based Smart Pathological Brain Detection System by Fractional Fourier Entropy. J Med Syst, 2016. 40(7): p. 173

[29]Siddiqui, M.F., et al., An Automated and Intelligent Medical Decision Support System for Brain MRI Scans Classification. Plos One, 2015. 10(8), Article ID: e0135875

[30]Liu, G., Computer-aided diagnosis of abnormal breasts in mammogram images by weighted-type fractional Fourier transform. Advances in Mechanical Engineering, 2016. 8(2), Article ID: 11

[31]Wu, X., Smart detection on abnormal breasts in digital mammography based on contrast-limited adaptive histogram equalization and chaotic adaptive real-coded biogeography-based optimization. Simulation, 2016. 92(9): pp. 873-885 